Int. J. Dev. Biol. 50: 323-332 (2006)

doi: $10.1387 /$ ijdb.052045lt

\title{
Biophysical mechanisms of cardiac looping
}

\author{
LARRY A. TABER* \\ Department of Biomedical Engineering, Washington University, St. Louis, MO, USA
}

\begin{abstract}
During early embryogenesis, the heart is a single, relatively straight tube which bends and twists (loops) rightward to create the basic plan of the mature heart. Despite intensive study for many decades, the biophysical mechanisms which drive and regulate cardiac looping have remained poorly understood. This review discusses, from a historical perspective, studies of looping mechanics and various theories which have been proposed for this complex process. Then, based on recently acquired data, a new biomechanical hypothesis is proposed for the first phase of looping (c-looping). Understanding morphogenetic mechanisms would facilitate research devoted to preventing and treating congenital heart malformations caused by looping abnormalities.
\end{abstract}

KEY WORDS: cardiac morphogenesis, heart development, left-right asymmetry, biomechanics

\section{Introduction}

The heart is the first functioning organ in the embryo. In the human embryo, cardiac contractions begin about 17 days postconception, when the heart is essentially a single, relatively straight, muscle-wrapped tube. Soon after the initial contractions, the heart tube bends and twists (loops) into a curved tube with its convex surface normally directed toward the right side of the embryo. Cardiac looping represents the first visible sign of leftright asymmetry in vertebrate embryos. When looping is complete, the heart has assumed the basic configuration necessary for further development into a four-chambered pump.

Because abnormal looping often leads to congenital heart defects, this process has received considerable attention over the years. Most investigators agree that heart development depends on both genetic and epigenetic factors, with the latter including the mechanochemical environment. Rapid progress is being made in defining the genetic and molecular factors that regulate looping (Brand, 2003; Harvey, 1998; Mercola and Levin, 2001; Srivastava and Olson, 1997), but the biophysical mechanisms that drive looping remain poorly understood (Männer, 2000; Taber et al., 1995). This review examines the role that biomechanics plays in the looping process.

This paper is organized as follows. First, morphological aspects of early heart development are described. Second, experimental results are summarized from studies that focus on looping mechanisms and the causes of left-right directionality. Next, various hypotheses that have been proposed for looping are discussed from a historical perspective. Lastly, we examine a new hypothesis in light of recent data.

\section{Early cardiac morphogenesis}

The chick embryo is a popular experimental model for studies of cardiac morphogenesis. Development of the chick heart parallels that of the human heart (DeHaan, 1967). Based on external characteristics of the developing embryo, the system of Hamburger and Hamilton (1951) divides the 21-day incubation period of the chick into 46 stages.

\section{Formation of the heart tube}

The heart arises from the mesoderm, a layer of tissue that lies between the endoderm and ectoderm in the early embryo (Linask, 2003; Yutzey and Kirby, 2002). In the chick at stage 5 (20 h), precardiac cells are located in mesodermal regions (cardiogenic plates) to the left and right sides of the embryo (Fig. 1A). Moving individually at first, these cells migrate anteriorly and toward the embryonic midline, possibly being guided by a spatially graded network of fibronectin located between the mesoderm and endoderm (Linask and Lash, 1986).

As the precardiac mesodermal cells migrate, N-cadherinmediated cell junctions form, binding the cells into a pair of bilateral epithelial sheets that take the form of an arch, with the head fold forming near the anterior end of the arch (Fig. 1B) (de la Cruz and Sanchez-Gomez, 1998; Linask, 2003; Trinh and Stainier, 2004). With little cell rearrangement occurring thereafter, the branches of this arch-shaped region continue to move

Abbreviations used in this paper: CJ, cardiac jelly; DM, dorsal mesocardium; ECM, extracellular matrix; EN, endocardium; MY, myocardium; OV, omphalomesenteric vein; SPL, splanchnopleure.

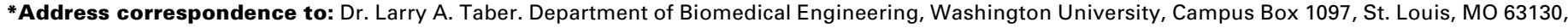
USA. Fax: +1-314-935-7448. e-mail: lat@wustl.edu
} 
laterally toward the midline, where they meet and fuse to form the heart tube (Fig. 1 C,D). As migration proceeds, the mesoderm splits into two layers - the somatic and splanchnic mesoderm which enclose the coelomic cavity (Patten, 1951). The somatic mesoderm and ectoderm comprise the somatopleure on the dorsal side of the embryo, while the splanchnic mesoderm and endoderm comprise the splanchnopleure (SPL) on the ventral side (Figs. 2,3A).

During stages 7-10 (24-36 h), the heart tube and primitive foregut form via folding and merging of the bilateral regions of the SPL in a cranial to caudal direction (Fig. 2). According to De Jong et al. (1990), once the precardiac mesoderm moves into its proper position on the dorsal side of the coelomic cavity, its component cells secrete extracellular matrix (ECM), which inflates this epithelium into a relatively straight but tapered tubular shape (about 200 $\mu \mathrm{m}$ in diameter) that is open along its dorsal side, i.e., the cross section has a U-shape ( $6 \mathrm{~S}$ and $10 \mathrm{~S}$ in Fig. 2B). Initially, the heart

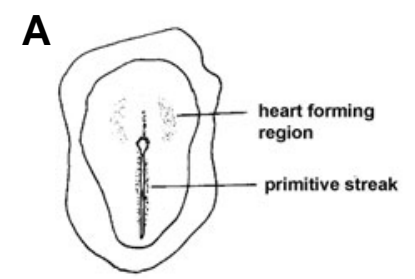

Stage 5

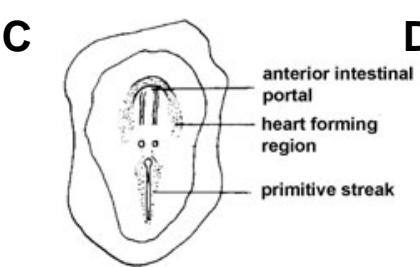

Stage 7

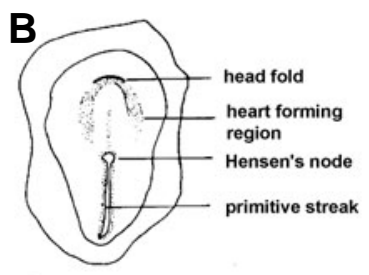

Stage 6

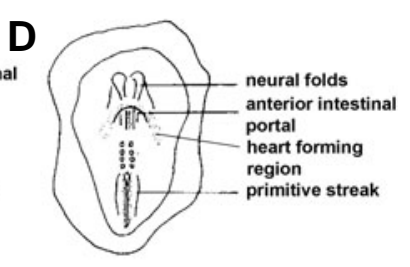

Stage 8
Fig. 1. Heart-forming region in chick embryo. Dotted areas show heart-forming region during stages 5-8 (from Linask and Lash, 1986). See text for details.

tube is composed of three layers (Fig. 3A): an inner layer of endocardium (EN), a relatively thick middle layer of ECM called cardiac jelly (CJ) and an outer layer of myocardium (MY), with the EN and MY being one-and two-cell-thick epithelia, respectively. Soon, the first myofibrils appear (Manasek et al., 1978), the first contractions occur (Hiruma and Hirakow, 1985) and looping begins (Patten, 1922; Romanoff, 1960), but effective blood flow does not start until stage 12 (1.9 days).

\section{Cardiac looping}

Looping begins at stage 10 , as the relatively straight heart tube bends and twists into a curved tube (Patten, 1922; Romanoff, 1960). Looping consists of two main phases: c-looping and slooping (Männer, 2000). During normal c-looping (stages 9-13, 30$50 \mathrm{~h}$ ), the heart transforms from a straight tube into a c-shaped tube (Fig. 3B) via two main deformation components: ventral bending and dextral (rightward) torsion ${ }^{1}$. This is seen clearly by labeling experiments (Fig. 4 A,B). Recently, Voronov et al. (2004) showed that a relatively small amount of dextral bending also occurs. It is important to note that, due to these deformations, the original ventral surface of the straight heart tube becomes the outer curvature (convex surface) of the looped heart, while the original dorsal side becomes the inner curvature (concave surface). During s-looping (stages 14-18, 52-68 h), the atrium moves superior to the ventricle, creating the basic final form of the heart (Fig. 3B). Later, during stages 21-36 (3.5-10 days), septation divides the tube into four chambers.

External constraints play a role in shaping the heart during looping. Initially, the heart tube is constrained by connections to the omphalomesenteric veins (OVs) at its caudal end and the outflow tract at its cranial end (Fig. 3B). In addition, a longitudinal structure called the dorsal mesocardium (DM) attaches the entire length of the heart to the embryo and the endodermal layer of the SPL lies against the ventral surface of the heart (Patten, 1951; Romanoff, 1960) (Fig. 3A). The OVs are created by the bilateral folds in the SPL as they continue to merge from head to foot, adding new segments to the caudal end of the heart tube (de la Cruz and Sanchez-Gomez, 1998). During looping, the dorsal side of the tube closes and the DM ruptures (by stage 13-, 2 days), leaving the heart attached to the embryo only at its ends. By stage 12, the heart tube is composed of a series of segments representing the primitive atrium, ventricle (future left and right ventricles) and conotruncus (Fig. 3B). A peristaltic contractile wave forces blood from the atrium through the ventricle and conotruncus.

Looping is a vital event during heart development, as even relatively minor looping perturbations can lead to serious structural defects. Clearly, bending and twisting the heart tube requires mechanical forces and researchers have shown that altering external and internally generated forces can affect looping (Itasaki et al., 1991; Manasek, 1976; Voronov et al., 2004; Voronov and Taber, 2002). Despite intensive study, however, many of the fundamental mechanisms that drive and regulate looping remain poorly understood.

\section{Studies of looping mechanisms}

Researchers have studied the biophysical mechanisms of looping for more than 80 years. In the following list, we summarize principal results from these studies.

\section{Characteristics of normal looping}

1. Looping begins when the first myofibrils appear (Manasek et al., 1978).

2. The DM always is located along the inner curvature of the looped ventricle, regardless of looping direction (Butler, 1952; Itasaki et al., 1991).

3. During c-looping, the MY thickens at the inner curvature of the heart tube and thins at the outer curvature (Manasek et al., 1972). 4. Before looping, actin microfilaments in the outer MY cell layer are arranged in a net-like pattern at cell borders (Itasaki et al., 1989), while circumferentially oriented actin bundles and striated myofibrils begin to form in the inner MY cell layer. During c-looping, circum-

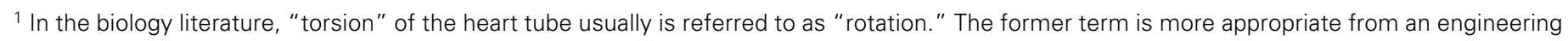

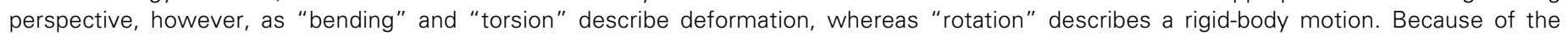
constraints at the ends of the heart tube, any rotation of the tube must be accompanied by torsion (twist).
} 
ferential actin remains near the inner curvature of the tube, but randomly oriented bundles appear near the outer curvature (Itasaki et al., 1989; Shiraishi et al., 1992). Changes in myocardial cell shape also follow this pattern (Manasek et al., 1984a).

5. During looping, the ECM protein flectin is expressed in higher concentrations on the left side of the tube and near the DM (Tsuda et al., 1996; Tsuda et al., 1998). However, the mechanistic effects of flectin are not yet known.

6 . There are no clear patterns of differential cell division or cell death in the looping heart (Stalsberg, 1969b; Stalsberg, 1970).

Effects of perturbing external loads and boundary conditions 7. The heart tube bends but does not twist when removed from the embryo and grown in culture (Butler, 1952; Manning and McLachlan, 1990). Moreover, when the stage $12-13$ chick heart is severed at either end, the heart loops as it does when isolated in culture, i.e., bending only (Flynn et al., 1991).

8. Removing the splanchnopleure greatly decreases the magnitude of torsion (rotation) during early c-looping (Voronov et al., 2004; Voronov and Taber, 2002).

9. Inhibiting cervical flexure of the embryo significantly affects the form of the heart during s-looping, as the atrium remains below the conotruncus (Männer et al., 1993).

\section{Effects of perturbing cells and ECM}

10. Inhibiting contractile protein synthesis with cycloheximide or bromodeoxyuridine, or inhibiting myofibrillogenesis with cytochalasin B prevents looping (Chacko and Joseph, 1974; Manasek, 1976; Manasek et al., 1978). Another study suggests, however, that looping does not require the presence of organized myofibrils (Koushik et al., 2001).

11. Globally disrupting actin filaments with high doses of cytochalasin B prevents or stops looping (Itasaki et al., 1991; Manasek, 1976). Inhibiting actin polymerization with relatively low concentrations of cytochalasin D or latrunculin A has similar effects (Latacha et al., 2005).

12. Arresting myocardial contraction with high potassium (Manasek and Monroe, 1972) or 2,3-butanedione monoxime (BDM) (unpublished results) does not prevent looping.

13. Inhibiting cytoskeletal contraction with the non-muscle myosin inhibitors Y-27632 and blebbistatin does not prevent looping in the chick (unpublished results), but Wei et al. (2001) found that $Y$ 27632 inhibits looping in the mouse. The reason for the apparent contradiction in these results is not clear.

14. Disrupting microtubules with colchicine causes MY cells to round up but does not affect c-looping (Icardo and Ojeda, 1984; Itasaki et al., 1991). However, the form of the heart is perturbed during s-looping (Icardo and Ojeda, 1984).

15. Dissolving the $\mathrm{CJ}$ with hyaluronidase does not inhibit looping (Baldwin and Solursh, 1989; Linask et al., 2003).

In summary, available data suggest that the bending component of c-looping is intrinsic to the heart tube and independent of function. In contrast, the torsional component of c-looping and the process of s-looping are influenced by external loads. Among the important structural elements in the heart tube are actin, flectin and the DM, but CJ pressure, cytoskeletal contraction and differential growth apparently have little or no effect.

\section{Studies of looping directionality}

Although vertebrates appear bilaterally symmetric on the outside, left-right asymmetry is the rule on the inside. The positioning of internal organs on either the left or right side of the body, as well as their orientation, is a highly conserved feature, with the normal asymmetric arrangement (situs solitus) occurring in well over $99 \%$ of humans. Interestingly, individuals who have all organs arranged in a mirror-image pattern (situs inversus) generally face no adverse physiological consequences. However, cardiac muscle fiber architecture in these people is reversed only in the basal region of the left ventricle, leading to abnormal (not just reversed) torsion during the cardiac cycle (Delhaas et al., 2004). In contrast, serious malformations may result if the heart is the only organ that is reversed (dextrocardia). For this reason, as well as to explore fundamental morphogenetic mechanisms of bilateral asymmetry, researchers long have been interested in the causes of left looping, which can lead to dextrocardia.

It is now known that looping directionality correlates with asymmetric expression of a number of genetic and molecular markers (Brand, 2003; Harvey, 1998; Mercola and Levin, 2001; Srivastava and Olson, 1997). However, as in other aspects of looping, the biophysical mechanisms that cause the heart to loop left or right are not well understood.
A

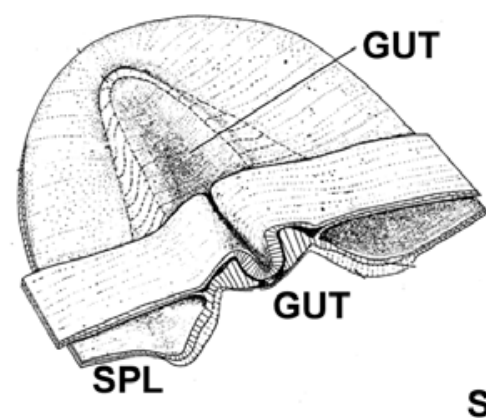

B

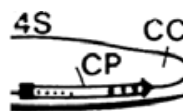

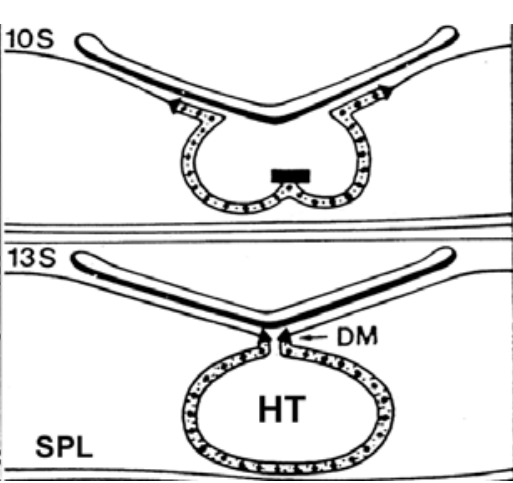

Fig. 2. Formation of heart tube. (A) 3D schematic of membrane folding to create the primitive foregut (GUT) in the chick embryo (from Bellairs, 1953) (B) Cross section showing temporal formation of the foregut and heart tube (From De Jong F. et al., 1990). Abbreviations: AlP, anterior intestinal portal; CC, coelomic cavity; CP, cardiogenic plate; DM, dorsal mesocardium; end, endoderm; HT, heart tube; S, somite 14S, stage 8; 8S, stage 9; 10S, stage 10; 13S, stage 11); SPL, splanchnopleure; VM, ventral mesocardium. 
Stalsberg (1970) used data from the literature to examine looping directionality in considerable detail. She studied a number of possibilities, including differential cell proliferation and death, differences in cell numbers contributed from the left and right cardiac primordia and other extrinsic and intrinsic asymmetries. Although many of Stalsberg's conclusions are valid, some of her analyses are based on the incorrect assumption that looping consists only of rightward bending (see next section). This misperception likely has caused many researchers to misinterpret some experimental results (Männer, 2000, 2004).

Intriguing experiments by Lepori (1967) and Itasaki et al. (1991) have been cited often as demonstrating the influence of extrinsic and intrinsic forces, respectively, on looping direction. In his study, Lepori (1967) found that cutting the SPL on the left side of the chick embryo does not change looping direction, but cutting it on the right side results in abnormal looping to the left. Itasaki et al. (1991), on the other hand, used crystals of cytochalasin B to disorganize actin in limited regions of the myocardium. Whereas disturbing actin on the left side of the heart had no apparent effect on looping direction, disruption on the right side in stage 8-9 chick embryos caused left looping in a significant number of hearts. Noting the circumferential orientation of actin filaments in the prelooped heart (Itasaki et al., 1989; Shiraishi et al., 1992), these investigators speculated that circumferential cytoskeletal contractile forces, normally stronger on the right side, pull the heart tube rightward, forcing it to rotate about the dorsal mesocardium, which initially anchors the tube to the embryo and acts as a pivot. If contraction on the right side is disrupted, then the contraction on the left side pulls the heart toward the left.

Although it seems clear that the SPL and actin microfilaments play major roles in the looping process (see next section), more recent work has questioned the results obtained by both Lepori (1967) and Itasaki et al. (1991). In particular, Voronov and Taber (2002; Voronov et al., 2004) have repeated both of these experiments, with similar results, but they found that the surface tension present in standard methods for culturing chick embryos can affect
2004). In addition, reversed left-right spatial distributions of the protein flectin in the precardiac mesoderm correlate with abnormal looping to the left (Linask et al., 2002; Linask et al., 2003; Tsuda et al., 1996; Tsuda et al., 1998), but the role played by flectin has not been determined.

Perhaps one of the most exciting findings in recent years is the discovery of an array of rapidly rotating monocilia located in the node (Nonaka et al., 1998), which is a signaling center in the early embryo (Gilbert, 2003). Normally, these cilia rotate clockwise in a vortical manner at approximately $600 \mathrm{rpm}$ and this rotary motion generates a flow directed toward the left (Nonaka et al., 1998; Nonaka etal., 2002; Okada etal., 1999). Recently, Cartwright etal. (2004) have analyzed this phenomenon using fluid mechanics. Notably, knocking out the cilia or their motility results in randomized looping direction (Nonaka et al., 1998; Okada et al., 1999) and artificially inducing rightward flow produces left looping. Speculation on the implications of these data includes the hypothesis that the flow deposits a morphogen on the left side of the node that initiates left-right asymmetry (Brueckner, 2001; Mercola, 2003; Nonaka et al., 1998; Supp et al., 2000).

\section{Previous hypotheses for looping}

Researchers long have speculated about the biophysical mechanisms that drive looping. Any valid hypothesis for looping must be consistent with the observations listed in the previous section.

In early work, Patten (1922) suggested that the heart tube is simply compelled to bend because its length grows faster than the space in which it is contained. This idea was later contradicted, however, by the finding that the heart tube bends when cultured in isolation (Butler, 1952; Manning and McLachlan, 1990). In addition, Patten (1922) speculated that the heart is forced to bend either leftward or rightward because of physical impediments supplied dorsally by the body of the embryo and ventrally by the yolk. But he could not explain why the heart almost always loops toward the right.
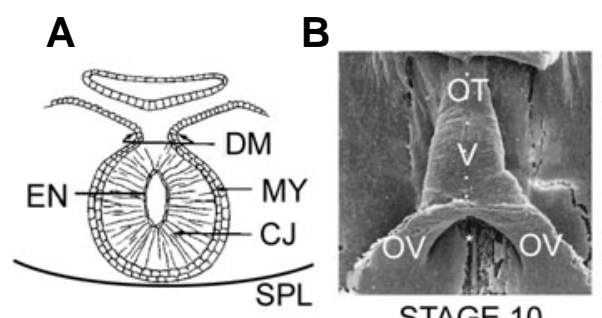

STAGE 10

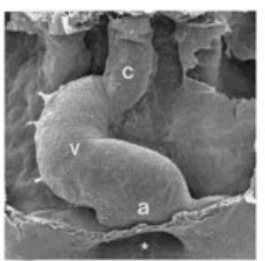

12

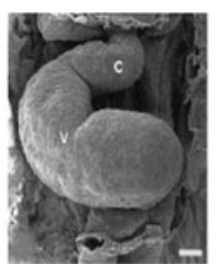

14

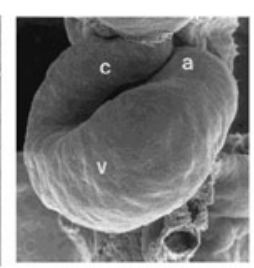

16
Fig. 3. Looping chick heart. (A) Schematic of cross section of stage 10 chick heart (from Manasek, 1983). CJ, cardiac jelly; DM, dorsal mesocardium; EN, endocardium; $M Y$, myocardium; SPL, splanchnopleure (B) Ventral views of looping chick heart (from Männer, 2000). a, atrium; c, conotruncus; OT, outflow tract; OV, omphalomesenteric veins; $V$ ventricle. looping morphology. When they conducted these experiments under a thin layer of fluid to eliminate surface tension, virtually no left looping occurred. Thus, the results of Lepori (1967) and Itasaki et al. (1991) may have been artifactual.

Much evidence suggests that the direction of looping is determined, at least in part, by events that occur prior to the formation of the heart tube. Studies have shown, for example, that inhibiting cell adhesion early in development can perturb looping direction. Blocking the function of the adhesion molecule $\mathrm{N}$-cadherin before stage 5 randomizes looping direction (Garcia-Castro et al., 2000), whereas degrading heparan sulfate to disrupt adhesion in the right precardiac area during stages 7-8 leads to left looping (Yue et al.,
Some investigators have speculated that hemodynamic forces mold the developing heart, with bending caused by blood impinging against the wall at the outer curvature of the heart tube (Spitzer and Lev, 1951). In fact, Hove et al. (2003) recently found that zebrafish hearts do not loop when inflow or outflow is blocked. The flow molding concept is contradicted, however, by three pieces of evidence. First, little flow passes through the heart when it bends in isolated culture. Second, looping progresses normally when the heartbeat is arrested by high potassium (Manasek and Monroe, 1972). Third, c-looping is essentially complete before effective flow begins at about stage 12 (Patten, 1951). The reasons for the results of Hove et al. (2003) are unclear. 
Another possibility is that differential growth causes the heart to bend, with cells at the outer curvature dividing faster than those at the inner curvature. However, Sissman (1966) found that cell multiplication rates in the looping heart show no consistent spatial pattern and Stalsberg (1969b; 1970) concluded that regional differences in cell division and cell death apparently are not great enough to produce significant bending. On the other hand, differential growth also could be caused by myocardial cells being added to opposite sides of the heart at different rates, or by cells
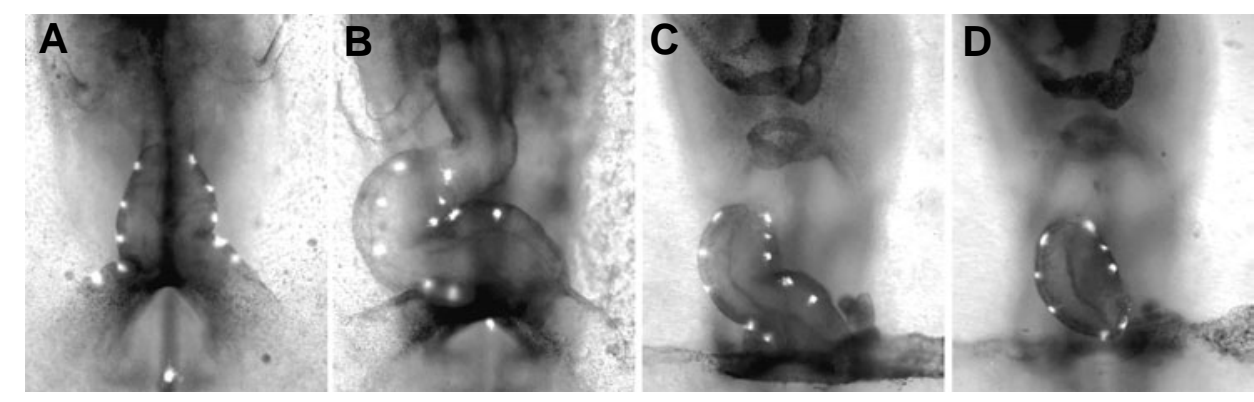

Fig. 4. Effect of splanchnopleure and omphalomesenteric veins on cardiac rotation (from Voronov et al., 2004). (A) Intact stage-10 heart with fluorescent labels along left and right sides. (B) Same heart at stage 11; as the heart rotates (twists), labels move toward dorsal and ventral sides of heart. (C) Splanchnopleure is removed; most rotation disappears within 1 hour. (D) Omphalomesenteric veins are severed; label positions indicate that remainder of torsion is lost, but heart remains bent slightly toward the right. moving from one region to another,

e.g., from the inner to the outer curvature. Some evidence suggests that the former mechanism contributes to looping, as relatively more cells are contributed to the right side of the cranial portion of the ventricle and to the left side of the caudal portion (DeHaan, 1967; Stalsberg, 1969a; Stalsberg, 1970). These findings, however, are more consistent with the relatively modest rightward bending described by Voronov et al. (2004), rather than the more pronounced ventral bending. Moreover, the myocardium is composed of tightly coupled cells during looping (Stalsberg and DeHaan, 1969) and we are not aware of direct evidence supporting a mechanism based on cell rearrangement. Therefore, available data do not appear to support differential growth as a major player in looping.

In a study of cell shape change in chick hearts, Manasek et al. (1972) found that myocardial cells at the outer curvature flatten during c-looping, while those near the inner curvature become thicker radially and longer circumferentially. They postulated that these cell shape changes are actively generated, possibly through myofibrillogenesis, to force the heart tube to bend. Later, however, they dropped this idea for unclear reasons in favor of a new hypothesis.

The new hypothesis put forth by the Manasek group was based on three main observations: (1) Cardiac jelly exerts an outward pressure on the myocardium and changes volume in response to changes in osmolarity of the surrounding media (Manasek et al., 1984a; Nakamura and Manasek, 1978). (2) The structure of the DM suggests that it provides a relatively stiff constraint along the dorsal side of the heart tube (Manasek et al., 1984b; Manasek, 1983). In fact, direct mechanical measurements now have confirmed that the inner curvature is much stiffer than other regions in the stage 12 chick heart (Zamir et al., 2003). (3) Myofibrillogenesis begins at about the same time that looping begins and inhibiting this process prevents looping (Manasek, 1976; Manasek et al., 1978). Integrating these observations, Manasek et al. (1984b) postulated that bending is caused by $\mathrm{CJ}$ pressure inflating the heart, with the DM constraining longitudinal extension and forcing the tube to bend with the DM along the inner curvature (see observation 2 above), while torsion is caused by wall stress being redirected through a helical system of myofibrils. According to this model, therefore, CJ pressure is the driving force for looping, with developing cardiomyocytes playing a passive, rather than active, role. Using a physical model constructed with a cylindrical latex membrane (the MY), tape (the DM) and a helix of stiffer material (myofibrils), these investigators showed that such a mechanism is physically plausible. They also suggested that regional differences in wall compliance can cause the observed bulging at the outer curvature. Unfortunately, this clever idea apparently was put to rest by Baldwin and Solursh (1989) and later by Linask et al. (2003), who found that digesting the $\mathrm{CJ}$ with hyaluronidase does not prevent looping.

So, by 1990, a number of hypotheses for looping had come and gone and most interest in the field turned toward the genetic side of the problem. However, even if the genetic and molecular factors involved in looping are clearly defined, there would still be a need to discover the link to specific morphogenetic mechanisms.

There are at least three difficulties that have compounded the looping problem. First, many researchers have not fully appreciated (or been aware of) the need to consider both ventral bending and dextral rotation in interpreting experimental results. [As a novice to looping, even this author was not immune to this (Taber et al., 1995).] Although this characteristic of c-looping has been known for many years (Butler, 1952), Männer (2000) felt a need to reemphasize it. And recently, Männer (2004) showed that the looped heart is actually a helix and that the sense of this helix in three-dimensional space must be considered to accurately describe looping morphology. In this light, repeating some prior experiments may be useful. Second, looping may involve a combination of several different mechanisms, some of which may be redundant and compensate when others fail (Stalsberg, 1970). While such redundancy would be advantageous to the embryo, it complicates matters considerably for the investigator. Third, visualizing the behavior of such a complex three-dimensional structure can be difficult (Männer, 2004) and physical intuition in such a nonlinear problem may be misleading.

Bending and torsion of a tube clearly involves mechanical forces. During the last decade, therefore, we have approached the looping problem from the perspective of a biomechanical engineer $^{2}$. Our work has involved experiments with chick embryos and

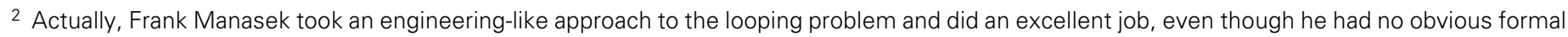
engineering training.
} 
computational models based on the principles of continuum mechanics. As described below, we now believe that the bending and torsional components of c-looping are driven by two different sets of forces: while bending is primarily a process intrinsic to the heart tube, torsion is driven by external loads.

\section{A new hypothesis for C-looping}

\section{Hypothesis for cardiac bending}

Currently, most investigators accept the view that bending is primarily a process intrinsic to the heart tube. Identifying the source of the force that drives cardiac bending, however, has remained elusive. It would seem logical that actin is involved, as this cytoskeletal protein plays a role in many morphogenetic processes (Alberts et al., 2002). Indeed, the cytochalasin B experiments of Manasek (1976) and Itasaki et al. (1991) are consistent with this idea. But what precisely is the role of actin? As discussed above, early work on looping had effectively ruled out differential growth and cell rearrangement. In 1990, two possibilities that remained were cytoskeletal contraction and actin polymerization.

Focusing on cardiac torsion and looping directionality, Itasaki et al. (1991) suggested that cytoskeletal contraction causes the heart to loop toward the right, but their results were later questioned (Voronov and Taber, 2002; Voronov et al., 2004). Recently, we explored this potential mechanism further by inhibiting contractile activity of non-muscle myosin II using ML-7, Y-27632 and blebbistatin (unpublished results). None of these drugs had an appreciable effect on overall looping morphology in whole embryo culture and the results were similar when these experiments were repeated on isolated hearts to focus on bending alone. The influence of these drugs on contractility was confirmed using regional stiffness measurements. Although the effects of ML-7 were relatively minor, hearts treated with Y-27632 and blebbistatin exhibited greatly decreased stiffness relative to controls, indicating loss of contractile force. Based on these results, we concluded that cytoskeletal contraction plays no significant role in looping.

To study the effects of actin polymerization, stage $9-11$ chick
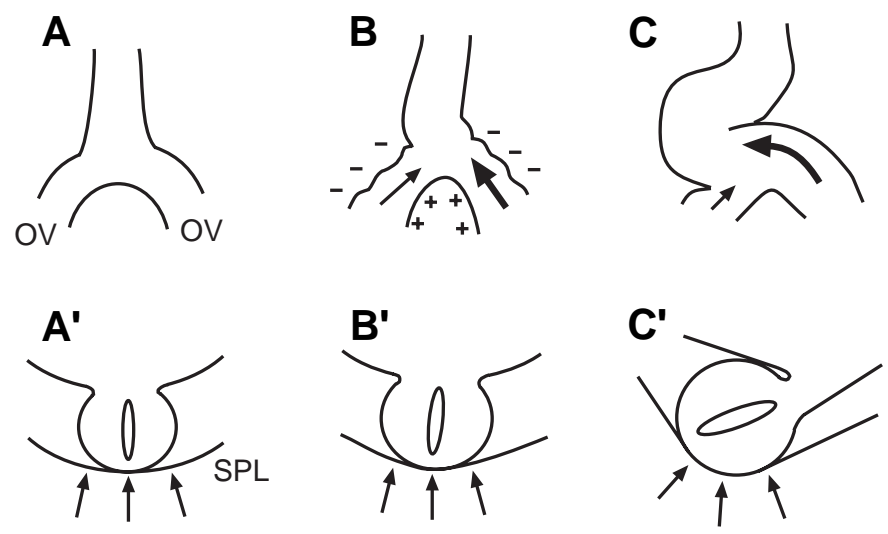

Fig. 5. Schematic of hypothesis for cardiac rotation. (courtesy Patrick Alford. $(A, B, C)$ Ventral view; $\left(A^{\prime}, B^{\prime}, C^{\prime}\right)$ cross sectional view. $\left(\mathbf{A}, \mathbf{A}^{\prime}\right)$ Straight heart tube before looping. (B, $\left.\mathbf{B}^{\prime}\right)$ Cytoskeletal contraction and cell migration cause compressive and tensile stresses along cranial and caudal surfaces, respectively, of omphalomesenteric veins (OV). Relatively greater force in left vein displaces caudal end of heart tube slightly toward the right. (C, $\left.\mathbf{C}^{\prime}\right)$ The splanchnopleure (SPL) pushes the heart tube dorsally, increasing rotation magnitude. hearts were exposed to cytochalasin D and latrunculin A (Latacha et al., 2005), which are more specific drugs than cytochalasin B for inhibiting actin polymerization (Fenteany and Zhu, 2003). In whole embryo culture, both drugs inhibited looping in a dose and stage dependent manner, similar to the results obtained by Manasek (1976) for cytochalasin B. Similar results were obtained for isolated hearts, but looping was affected at much lower doses. These data indicate that ongoing actin polymerization is required for cardiac bending during c-looping and, by process of elimination, we speculate that it provides the driving force.

If it can be determined that actin polymerization does indeed drive cardiac bending, we still must answer the following questions: (1) What is the specific mechanism by which actin polymerization causes the observed morphogenetic shape changes? (2) How are the forces integrated to bend the heart tube? (3) How are the forces regulated?

To answer the first question, we postulate that a growing cytoskeletal actin network causes changes in cell shape by exerting force against the cell membrane. In other words, we are essentially reviving the active cell-shape change idea of Manasek etal. (1972), which was subsequently dropped. This mechanism is similar to that which drives pseudopod extension in crawling cells (Alberts et al., 2002) and is supported by the prominent actin bundles seen during looping around myocardial cell borders (Itasaki et al., 1989).

To answer the second question, we examine the microstructural details of the developing myocardium. At the onset of looping, circumferentially aligned actin microfilaments are present in the myocardium (Itasaki et al., 1991; Manasek et al., 1984b; Shiraishi et al., 1992), but as the heart tube bends, actin fibers at the outer curvature become more randomly organized, while fibers near the inner curvature maintain their circumferential orientation (Itasaki et al., 1989). Polymerization in these predominant fiber directions (isotropic on the ventral side and circumferential on the dorsal side) would cause cells near the outer curvature (ventral side) to become flatter in the radial direction and longer in the circumferential and longitudinal directions, whereas cells near the inner curvature (dorsal side) would become longer circumferentially but shorter longitudinally (to preserve cell volume). These changes in cell shape are consistent with those observed by Manasek etal. (1972) and, as discussed later, a computational model has shown that they can cause the observed bending.

The third question is more difficult to answer. Developmental genes likely play a role in regulating morphogenetic forces. In the heart, they may dictate actin geometry, which then defines the subsequent changes in cell shape. The signal to stop bending also may be genetic, or it could be controlled by constraints such as the gradual accumulation of longitudinally oriented myofilaments, which would stiffen cells and make the heart tube harder to bend, as suggested by Nakamura et al. (1980). Another possible regulatory mechanism is mechanical feedback, which has been studied in other developing systems by Beloussov (1998). Regulation of the looping process remains an open question that warrants future study.

To our knowledge, actin polymerization has not been suggested previously as a cause of largescale morphogenetic shape changes. Moreover, one may question whether growing actin filaments can exert forces great enough to bend the heart tube. In fact, various researchers have estimated that polymerization stresses can be 

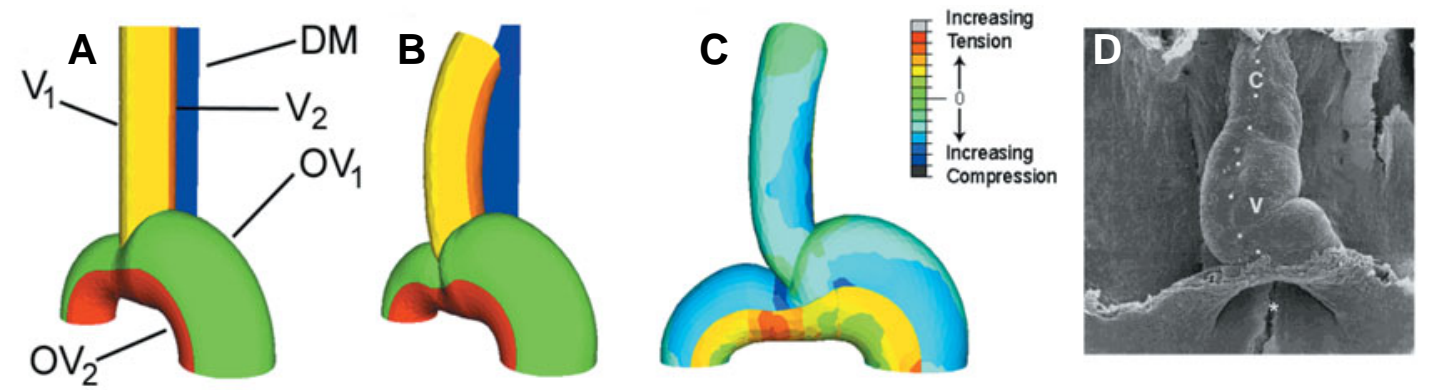

Fig. 6. Finite element model for looping heart (from Voronov et al., 2004). (A) Oblique view of undeformed model showing specified morphogenetic forces (DM, dorsal mesocardium; V1, ventral half of ventricle - longitudinal-circumferential actin poly-

merization; V2, dorsal half of ventricle - circumferential polymerization; OV1, cranial half of omphalomesenteric veins - longitudinal growth (cell influx); OV2, caudal half of omphalomesenteric veins - longitudinal contraction). (B) Diagonal view showing ventral bending. (C) Ventral view showing rightward rotation (colors indicate longitudinal stresses). (D) Stage-10+chick heart for comparison with (C) (from Männer, 2000).

as large as a few hundred pascals (Howard, 2001; Upadhyaya et al., 2003). According to material property measurements of stage 12 chick hearts, such stresses can indeed produce myocardial deformations large enough (> 50\% strain) to cause significant bending of the heart tube (Zamir and Taber, 2004a; Zamir and Taber, 2004b).

\section{Hypothesis for cardiac torsion}

Some investigators have speculated that torsion, like bending, is caused by internal forces (Itasaki et al., 1991; Manasek et al., 1984b). Recent work has shown, however, that the SPL and OVs influence torsion during early c-looping (Voronov et al., 2004; Voronov and Taber, 2002). For example, when the SPL is removed from a stage-11 chick embryo, the heart immediately springs back partially toward the midline of the embryo and then slowly untwists until most of the torsion is lost (Fig. $4 \mathrm{~B}, \mathrm{C}$ ). If the OVs then are severed, the remainder of the twist disappears, with the ventricle remaining bent slightly toward the right (Fig. 4D). In addition, when the SPL is removed from stage 10 embryos, the heart bends ventrally but rotates little when cultured for six hours (Voronov and Taber, 2002). Hence, external forces supplied by the SPL and OVs appear to play prominent roles in the torsional component of clooping. As described below, we speculate that the OVs force the heart tube slightly rightward and then the SPL pushes it dorsally and farther rightward as the tube rotates about the DM.

\section{Integrated hypothesis for C-looping}

Taken together, the results of these and other studies suggest the following working hypothesis for the mechanism for c-looping (see Fig. 5; Voronov et al., 2004):

1. Beginning at stage 10 (in the chick), precardiac cells in the caudal regions of the OVs undergo longitudinal cytoskeletal contraction. This generates tensile and compressive stresses in the caudal and cranial parts of the veins, respectively, as found by Voronov et al. (2004) (Fig. 5B). These stresses pull precardiac mesoderm through the veins toward the caudal end of the heart, possibly inducing additional influx of cells through active migration along lines of stress-aligned fibronectin (Linask and Lash, 1986; Linask and Lash, 1988a; Linask and Lash, 1988b; Toyoizumi et al., 1991; Trinh and Stainier, 2004).

2. Incoming cells in both OVs push against the caudal end of the heart tube and fuse to form the primitive left ventricle and atrium. Due possibly to asymmetric vein geometry or contractile strength, the left vein normally pushes with greater force, displacing the heart slightly toward the right (Fig. 5B). The DM constrains this displacement, converting it into a small rightward rotation about the relatively fixed dorsal side of the heart (Fig. 5B').

3 . The heart bends ventrally via actin polymerization and the SPL exerts a compressive force against the ventral surface of the ventricle, pushing it farther toward the right (Fig. 5 C,C'). Further bending and torsion causes the DM to rupture, allowing the heart to deform into a c-shaped tube.

We also speculate that redundant asymmetries, besides that supplied by the OV forces, bias the heart to loop toward the right side. Rightward bending of the tube (Voronov etal., 2004) and the rightward shifting of the conotruncus (Männer, 2000) are two candidates. Thus, if one asymmetry is disrupted, others still can induce normal dextral looping.

To test whether this hypothesis is physically plausible, Voronov et al. (2004) developed a computational model for the stage 10 heart that includes the OVs and the DM (Fig. 6A). Consistent with the morphology (Fig. 3B), the left vein had a larger diameter than the right vein. And consistent with observed changes in actin geometry and MY cell shape during c-looping (Itasaki et al., 1989; Itasaki etal., 1991; Manasek etal., 1984b; Manasek etal., 1984a; Manasek et al., 1972; Shiraishi et al., 1992), actin polymerization was specified in the circumferential direction over the dorsal half of the ventricle and in the circumferential-longitudinal directions over the ventral half. In the OVs, longitudinal contraction was prescribed for the lower halves of the veins, while the upper
Fig. 7. Effects on looping of removing splanchnopleure and right omphalomesenteric vein (from Voronov et al., 2004). (A,D) Stage-10 heart and idealized model. (B,E) Heart and model immediately after removing vein. (C,F) Heart and model $18 \mathrm{~h}$ later; in agreement with observed geometry, the model ventricle first jogs leftward (E) and then deforms rightward (F).
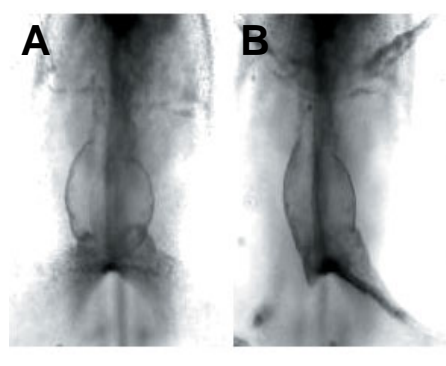
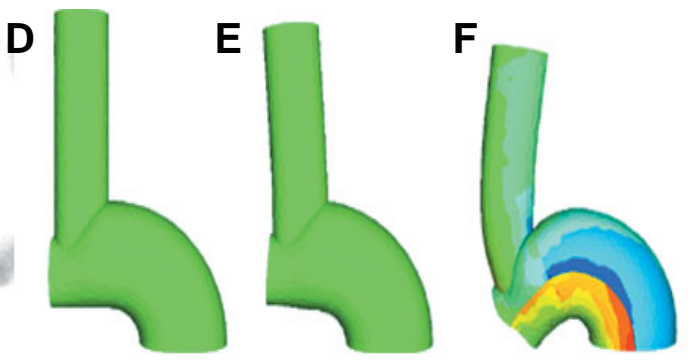
halves grew longitudinally to simulate the influx of cells into the heart. When these forces were turned on, the heart tube bent ventrally (Fig. 6B) and displaced and rotated slightly toward the right (Fig. 6C). The resulting geometries of the heart and veins resemble those of the stage $10+$ chick embryo (Fig. 6D) and the tensile and compressive stresses in the lower and upper parts of the OVs, respectively, agree with measured stress data.

To test of the predictive capability of this model, Voronov et al. (2004) removed the right OV in stage-10 embryos and observed that the ventricle first moved slightly to the left and then was pushed rightward by the remaining OV during the next $18 \mathrm{~h}$ in culture (Fig. 7 A,B,C). With the right vein removed and the same model parameters used for normal looping, the model predicted morphology that is remarkably similar to the experimental heart (Fig. 7 D,E,F). According to this model, tension in the left vein initially pulls the heart to the left and then cell migration pushes the heart slowly rightward. When the left vein was cut, the experimental and computed deformations were similar but opposite in direction.

Finally, according to our hypothesis, reducing the force in the left OV below that exerted by the right OV should result in looping to the left. Indeed, when Voronov et al. (2004) removed a portion of the left vein while leaving the SPL mostly intact, more than $25 \%$ of these operations produced left looping, which rarely occurs under normal culture conditions.

\section{Future directions}

While aspects of the mechanics of looping are now becoming clear, much remains unsettled. For example, although some mechanical forces involved in looping have been identified, it remains to be established how these forces are regulated spatially and temporally to produce a looped heart tube. Further investigation is needed to determine whether the proposed actin polymerization mechanism can produce the necessary changes in tissue shape on a global scale. In addition, the possible roles played by redundant mechanisms have not been elucidated. Finally, the mechanisms of s-looping have received relatively little attention, as have the mechanisms involved in the initial creation of the heart tube. The latter may have implications in left-right looping directionality.

In the long term, gaining a complete understanding of heart development requires finding the link between gene expression and morphomechanics (Brouzes and Farge, 2004). Reaching this goal will require the combined expertise of developmental biologists and biomechanical engineers. Toward this end, the pioneering studies of Huang et al. (2001b;2001a) may provide a valuable roadmap.

\section{Acknowledgements}

The author wishes to thank colleagues (Professors Renato Perucchio and Elliot L. Elson), postdocs (Drs. Dmitry A. Voronov, Kimberly S. Latachaand Ashok Ramasubramanian) andstudents (Mathieu C. Rémond, Dr. Evan A. Zamir, Patrick W. Alford and Amy Y. Chen) who contributed directly to the work presented in this review. This research was supported by NIH grant R01 HL64347. Finally, we acknowledge that several figures in this paper are reprinted with permission from Elsevier (Figs. 1, 4, 6 A$C$ and 7), Wiley Interscience (Figs. 2B, $3 B$ and 6D), the Company of Biologists (Fig. 2A) and the Novartis Foundation (Fig. 3A).

\section{References}

ALBERTS,B., JOHNSON,A., LEWIS,J., RAFF,M., ROBERTS,K. and WALTER,P. (2002). Molecular Biology of the Cell. New York: Garland.

BALDWIN,H.S. and SOLURSH,M. (1989). Degradation of hyaluronic acid does not prevent looping of the mammalian heart in situ. Dev. Biol. 136: 555-559.

BELLAIRS,R. (1953). Studies on the development of the foregut in the chick blastoderm. 1. The presumptive foregut area. J. Embryol. Exp. Morph. 1:115124.

BELOUSSOV,L.V. (1998). The Dynamic Architecture of a Developing Organism: An Interdisciplinary Approach to the Development of Organisms. Dordrecht, the Netherlands: Kluwer.

BRAND, T. (2003). Heart development: molecular insights into cardiac specification and early morphogenesis. Dev. Biol. 258: 1-19.

BROUZES,E. and FARGE,E. (2004). Interplay of mechanical deformation and patterned gene expression in developing embryos. Curr. Opin. Genet. Dev. 14: 367-374.

BRUECKNER,M. (2001). Cilia propel the embryo in the right direction. Am. J. Med. Genet. 101: 339-344.

BUTLER,J.K. (1952). An experimental analysis of cardiac loop formation in the chick. M.S. Thesis, University of Texas.

CARTWRIGHT,J.H., PIRO,O. and TUVAL,I. (2004). Fluid-dynamical basis of the embryonic development of left-right asymmetry in vertebrates. Proc. Natl. Acad. SCi. USA 101: 7234-7239.

CHACKO,S. and JOSEPH,X. (1974). The Effect of 5-Bromodeoxyuridine (BrdU) on Cardiac Muscle Differentiation. Dev. Biol. 40: 340-354.

DE JONG, F., GEERTS,W.J., LAMERS,W.H., LOS,J.A. and MOORMAN,A.F. (1990). Isomyosin expression pattern during formation of the tubular chicken heart: a three-dimensional immunohistochemical analysis. Anat. Rec. 226:213227.

DE LA CRUZ,M.V. and SANCHEZ-GOMEZ,C. (1998). Straight heart tube. Primitive cardiac cavities vs. primitive cardiac segments. In Living Morphogenesis of the Heart. (ed. de la Cruz,M.V. and Markwald,R.R.), pp. 85-98. Boston: Birkhauser.

DEHAAN,R.L. (1967). Development of form in the embryonic heart. An experimental approach. Circulation 35: 821-833.

DELHAAS,T., DECALUWE,W., RUBBENS,M., KERCKHOFFS,R. and ARTS,T. (2004). Cardiac fiber orientation and the left-right asymmetry determining mechanism. Ann. N. Y. Acad. Sci. 1015: 190-201.

FENTEANY,G. and ZHU,S. (2003). Small-molecule inhibitors of actin dynamics and cell motility. Curr. Top. Med. Chem. 3: 593-616.

FLYNN,M.E., PIKALOW,A.S., KIMMELMAN,R.S. and SEARLS,R.L. (1991). The mechanism of cervical flexure formation in the chick. Anat. Embryol. 184: 411420.

GARCIA-CASTRO,M.I., VIELMETTER,E. and BRONNER-FRASER,M. (2000). NCadherin, a cell adhesion molecule involved in establishment of embryonic leftright asymmetry. Science 288: 1047-1051.

GILBERT,S.F. (2003). Developmental Biology. Sunderland, MA: Sinauer Associates.

HAMBURGER,V. and HAMILTON,H.L. (1951). A series of normal stages in the development of the chick embryo. J. Morphol. 88: 49-92.

HARVEY,R.P. (1998). Cardiac looping - an uneasy deal with laterality. Semin. Cell Dev. Biol. 9: 101-108.

HIRUMA,T. and HIRAKOW,R. (1985). An ultrastructural topographical study on myofibrillogenesis in the heart of the chick embryo during pulsation onset period. Anat. Embryol. 172: 325-329.

HOVE,J.R., KOSTER,R.W., FOROUHAR,A.S., ACEVEDO-BOLTON,G., FRASER,S.E. and GHARIB,M. (2003). Intracardiac fluid forces are an essential epigenetic factor for embryonic cardiogenesis. Nature 421: 172-177.

HOWARD,J. (2001). Mechanics of Motor Proteins and the Cytoskeleton. Sunderland, MA: Sinauer.

HUANG,W., SHER,Y.P., GADO-WEST,D., WU,J.T., PECK,K. and FUNG,Y.C. (2001a). Tissue remodeling of rat pulmonary artery in hypoxic breathing. I. Changes of morphology, zero-stress state and gene expression. Ann. Biomed. Eng 29: 535-551. 
HUANG,W., SHER,Y.P., PECK,K. and FUNG,Y.C. (2001b). Correlation of gene expression with physiological functions: Examples of pulmonary blood vessel rheology, hypoxic hypertension and tissue remodeling. Biorheology38: 75-87.

ICARDO,J.M. and OJEDA,J.L. (1984). Effects of colchicine on the formation and looping of the tubular heart of the embryonic chick. Acta Anat. 119: 1-9.

ITASAKI,N., NAKAMURA,H., SUMIDA,H. and YASUDA,M. (1991). Actin bundles on the right side in the caudal part of the heart tube play a role in dextro-looping in the embryonic chick heart. Anat. Embryol. 183: 29-39.

ITASAKI,N., NAKAMURA,H. and YASUDA,M. (1989). Changes in the arrangement of actin bundles during heart looping in the chick embryo. Anat. Embryol. 180: 413-420.

KOUSHIK,S.V., WANG,J., ROGERS,R., MOSKOPHIDIS,D., LAMBERT,N.A., CREAZZO,T.L. and CONWAY,S.J. (2001). Targeted inactivation of the sodiumcalcium exchanger (Ncx1) results in the lack of a heartbeat and abnormal myofibrillar organization. FASEB J. 15: 1209-1211.

LATACHA, K. S., REMOND, M., RAMASUBRAMANIAN, A., CHEN, A. Y., ELSON, E. L. and TABER, L. A. (2005). The role of actin polymerization in bending of the early heart tube. Dev. Dyn. 233: 1272-1286.

LEPORI,N.G. (1967). Research on heart development in chick embryo under normal and experimental conditions. Monit. Zool. Ital. 1: 159-183.

LINASK,K.K. (2003). Regulation of heart morphology: current molecular and cellular perspectives on the coordinated emergence of cardiac form and function. Birth Defects Res. Part C Embryo. Today 69: 14-24.

LINASK,K.K., HAN,M.D., LINASK,K.L., SCHLANGE,T. and BRAND,T. (2003). Effects of antisense misexpression of CFC on downstream flectin protein expression during heart looping. Dev. Dyn. 228: 217-230.

LINASK,K.K. and LASH,J.W. (1986). Precardiac cell migration: fibronectin localization at mesoderm-endoderm interface during directional movement. Dev. Biol. 114: 87-101

LINASK,K.K. and LASH,J.W. (1988a). A role for fibronectin in the migration of avian precardiac cells. I. Dose-dependent effects of fibronectin antibody. Dev. Biol. 129: 315-323.

LINASK,K.K. and LASH,J.W. (1988b). A role for fibronectin in the migration of avian precardiac cells. II. Rotation of the heart-forming region during different stages and its effects. Dev. Biol. 129: 324-329.

LINASK,K.K., YU,X., CHEN,Y. and HAN,M.D. (2002). Directionality of heart looping: effects of Pitx2c misexpression on flectin asymmetry and midline structures. Dev. Biol. 246: 407-417.

MANASEK,F.J. (1976). Heart development: interactions involved in cardiac morphogenesis. In Cell Surface in Animal Embryogenesis and Development. (ed. Poste,G. and Nicholson,G.L.), pp. 545-598. New York: North-Holland.

MANASEK,F.J. (1983). Control of early embryonic heart morphogenesis: a hypothesis. In Development of the Vascular System. (ed. Nugent,J. and O'Connor,M.), pp. 4-19. London: Pitman.

MANASEK,F.J., BURNSIDE,M.B. and WATERMAN,R.E. (1972). Myocardial cell shape changes as a mechanism of embryonic heart looping. Dev. Biol. 29: 349371.

MANASEK,F.J., ISOBE,Y., SHIMADA,Y. and HOPKINS,W. (1984a). The embryonic myocardial cytoskeleton, interstitial pressure and the control of morphogenesis. In Congenital Heart Disease: Causes and Processes. (ed. Nora,J.J. and Takao,A.), pp. 359-376. Mount Kisco, NY: Futura Publishing.

MANASEK,F.J., KULIKOWSKI,R.R. and FITZPATRICK,L. (1978). Cytodifferentiation: A Causal Antecedent of Looping? Birth Defects 14: 161-178.

MANASEK,F.J., KULIKOWSKI,R.R., NAKAMURA,A., NGUYENPHUC,Q. and LACKTIS,J.W. (1984b). Early heart development: a new model of cardiac morphogenesis. In Growth of the Heart in Health and Disease. (ed. Zak,R.), pp. 105-130. New York: Raven Press.

MANASEK,F.J. and MONROE,R.G. (1972). Early cardiac morphogenesis is independent of function. Dev. Biol. 27: 584-588.

MÄNNER,J. (2000). Cardiac looping in the chick embryo: a morphological review with special reference to terminological and biomechanical aspects of the looping process. Anat. Rec. 259: 248-262.

MÄNNER,J. (2004). On rotation, torsion, lateralization and handedness of the embryonic heart loop: new insights from a simulation model for the heart loop of chick embryos. Anat. Rec. 278A: 481-492.
MÄNNER,J., SEIDL,W. and STEDING,G. (1993). Correlation between the embry onic head flexures and cardiac development. Anat. Embryol. 188: 269-285.

MANNING,A. and MCLACHLAN,J.C. (1990). Looping of chick embryo hearts in vitro. J. Anat. 168: 257-263.

MERCOLA,M. (2003). Left-right asymmetry: nodal points. J. Cell Sci. 116: 32513257.

MERCOLA,M. and LEVIN,M. (2001). Left-right asymmetry determination in vertebrates. Annu. Rev. Cell Dev. Biol. 17: 779-805.

NAKAMURA,A., KULIKOWSKI,R.R., LACKTIS,J.W. and MANASEK,F.J. (1980). Heart looping: a regulated response to deforming forces. In Etiology and Morphogenesis of Congenital Heart Disease. (ed. van Praagh,R. and Takao,A.), pp. 81-98. Mount Kisco, NY: Futura Publishing.

NAKAMURA,A. and MANASEK,F.J. (1978). Experimental Studies of the Shape and Structure of Isolated Cardiac Jelly. J. Embryol. Exp. Morph. 43: 167-183.

NONAKA,S., SHIRATORI,H., SAIJOH,Y. and HAMADA,H. (2002). Determination of left-right patterning of the mouse embryo by artificial nodal flow. Nature 418: 96-99.

NONAKA,S., TANAKA, Y., OKADA, Y., TAKEDA,S., HARADA,A., KANAI,Y., KIDO,M. and HIROKAWA,N. (1998). Randomization of left-right asymmetry due to loss of nodal cilia generating leftward flow of extraembryonic fluid in mice lacking KIF3B motor protein. Cel/95: 829-837.

OKADA,Y., NONAKA,S., TANAKA,Y., SAIJOH,Y., HAMADA,H. and HIROKAWA,N (1999). Abnormal nodal flow precedes situs inversus in iv and inv mice. Mol. Cell 4: 459-468.

PATTEN,B.M. (1951). Early Embryology of the Chick. New York: McGraw-Hill.

PATTEN,B.M. (1922). The Formation of the Cardiac Loop in the Chick. Am. J. Anat. 30: 373-397.

ROMANOFF,A.L. (1960). The Avian Embryo: Structural and Functional Development. New York: Macmillan.

SHIRAISHI,I., TAKAMATSU,T., MINAMIKAWA,T. and FUJITA,S. (1992). 3-D observation of actin filaments during cardiac myofibrinogenesis in chick embryo using a confocal laser scanning microscope. Anat. Embryol. 185: 401-408.

SISSMAN,N.J. (1966). Cell multiplication rates during development of the primitive cardiac tube in the chick embryo. Nature 210: 504-507.

SPITZER,A. and LEV,M. (1951). The Architecture of Normal and Malformed Hearts. Springfield, III: Thomas.

SRIVASTAVA,D. and OLSON,E.N. (1997). Knowing in your heart what's right. Trends Cell Biol. 7: 447-453.

STALSBERG,H. (1969a). The origin of heart asymmetry: right and left contributions to the early chick embryo heart. Dev. Biol. 19: 109-127.

STALSBERG,H. (1969b). Regional mitotic activity in the precardiac mesoderm and differentiating heart tube in the chick embryo. Dev. Biol. 20: 18-45.

STALSBERG,H. (1970). Mechanism of dextral looping of the embryonic heart. Am. J. Cardiol. 25: 265-271.

STALSBERG,H. and DEHAAN,R.L. (1969). The precardiac areas and formation of the tubular heart in the chick embryo. Dev. Biol. 19: 128-159.

SUPP,D.M., POTTER,S.S. and BRUECKNER,M. (2000). Molecular motors: the driving force behind mammalian left-right development. Trends Cel/Biol. 10:4145.

TABER,L.A., LIN,I.E. and CLARK,E.B. (1995). Mechanics of cardiac looping. Dev. Dyn. 203: 42-50.

TOYOIZUMI,R., SHIOKAWA,K. and TAKEUCHI,S. (1991). The behavior and cytoskeletal system of chick gastrula mesodermal cells on substrata coated with lines of fibronectin. J. Exp. Zool. 260: 345-353.

TRINH,L.A. and STAINIER,D.Y. (2004). Fibronectin regulates epithelial organization during myocardial migration in zebrafish. Dev. Ce//6: 371-382.

TSUDA,T., MAJUMDER,K. and LINASK,K.K. (1998). Differential expression of flectin in the extracellular matrix and left-right asymmetry in mouse embryonic heart during looping stages. Dev. Genetics 23: 203-214.

TSUDA,T., PHILP,N., ZILE,M.H. and LINASK,K.K. (1996). Left-right asymmetric localization of flectin in the extracellular matrix during heart looping. Dev. Biol. 173: $39-50$

UPADHYAYA,A., CHABOT,J.R. andREEVA,A., SAMADANI,A. and VAN OUDENAARDEN,A. (2003). Probing polymerization forces by using actin- 
propelled lipid vesicles. Proc. Natl. Acad. Sci. USA 100: 4521-4526.

VORONOV,D.A., ALFORD,P.W., XU,G. and TABER,L.A. (2004). The role of mechanical forces in dextral rotation during cardiac looping in the chick embryo. Dev. Biol. 272: 339-350.

VORONOV,D.A. and TABER,L.A. (2002). Cardiac looping in experimental conditions: the effects of extraembryonic forces. Dev. Dyn. 224: 413-421.

WEI,L., ROBERTS,W., WANG,L., YAMADA,M., ZHANG,S., ZHAO,Z., RIVKEES,S.A., SCHWARTZ,R.J. and IMANAKA-YOSHIDA,K. (2001). Rho kinases play an obligatory role in vertebrate embryonic organogenesis. Deve/opment 128: 2953-2962.

YUE,X., SCHULTHEISS,T.M., MCKENZIE,E.A. and ROSENBERG,R.D. (2004).
Role of heparan sulfate in dextral heart looping in chick. Glycobiology 14: 745755.

YUTZEY,K.E. and KIRBY,M.L. (2002). Wherefore heart thou? Embryonic origins of cardiogenic mesoderm. Dev. Dyn. 223: 307-320.

ZAMIR,E.A., SRINIVASAN,V., PERUCCHIO,R. and TABER,L.A. (2003). Mechanical asymmetry in the embryonic chick heart during looping. Ann. Biomed. Eng 31: 1327-1336.

ZAMIR,E.A. and TABER,L.A. (2004a). Material properties and residual stress in the stage 12 chick heart during cardiac looping. J. Biomech. Eng. 126: 823-830.

ZAMIR,E.A. and TABER,L.A. (2004b). On the effects of residual stress in microindentation tests of soft tissue structures. J. Biomech. Eng. 126: 276-283. 